\section{A ORGANIZAÇÃO POLÍTICO-CIENTÍFICA NOS EUA NOS ANOS DE 1960 E SEU IMPACTO PARA A DIMENSÃO ACADÊMICA DA EDUCAÇÃO FíSICA}

\author{
THE POLITICAL-SCIENTIFIC ORGANIZATION IN THE USA IN THE 1960S AND \\ ITS IMPACT ON THE ACADEMIC DIMENSION OF PHYSICAL EDUCATION
}

\author{
LA ORGANIZACIÓN POLÍTICA Y CIENTÍFICA EN LOS EE.UU. EN LA DÉCADA \\ DE 1960 Y SU IMPACTO EN LA DIMENSIÓN ACADÉMICA DE LA EDUCACIÓN \\ FISICA
}

Anísio Calciolari Junior*, Jeane Barcelos Soriano*
Palavras-chave Política nacional de ciência, tecnologia e inovação. Instituições acadêmicas: organização \& administração. História. Estados Unidos.

\section{Keywords:} National science, technology and innovation policy. Schools: organization \& administration. History. United States.

\section{Palabras clave} Política nacional de ciencia, tecnología e innovación. Instituciones académicas: organización \& administración. Historia. Estados Unidos.
Resumo: $O$ objetivo desse ensaio foi identificar como mudanças políticas do campo científico nos Estados Unidos (EUA) podem ter influenciado a organização da área de conhecimento da Educação Física norte-americana a partir da década de 1960. Destacaremos eventos históricos no campo político-científico dos EUA após a Segunda Guerra Mundial que estabeleceram uma nova relação entre Estado, Ciência e Universidade. A Educação Física sofre os impactos das novas regras dos campos político e científico, e vê a necessidade de se reorganizar, deflagrando uma série de ações e movimentos políticos com o objetivo de se manter na Universidade.

Abstract: The objective of this essay is to identify how the political changes of the scientific field in the United States (USA) may have influenced the organization of the knowledge area of the American Physical Education (PE) from the 1960s. We point historical events in the political-scientific field in the USA after the World War II that established a new relation among State, Science and University. The Physical Education has suffered the impacts of these new rules in the scientific and political fields, noticing the necessity to readapt through a series of political movements aiming to maintain itself at the University.

Resumen: El objetivo de este estudio fue identificar cómo los cambios políticos del ámbito científico en los Estados Unidos (EE.UU.) pueden haber influido en la organización del área de conocimiento de la Educación Física estadunidense de la década de 1960. Destacaremos los acontecimientos históricos en el campo políticocientífico de los EE.UU. después de la Segunda Guerra Mundial que estableció una nueva relación entre el Estado, la Ciencia y la Universidad. La Educación Física sufrió los impactos de las nuevas reglas de los campos político y científico, y ve la necesidad de reorganizar, lo que provocó una serie de acciones y movimientos políticos con el objetivo de mantenerse en la Universidad
*Universidade Estadual de Londrina. Londrina, PR, Brasil.

E-mail: anisiocalciolari@ hotmail.com

Recebido em: 25.6.2014

Aprovado em: 23.3.2015

(c) (i) () Licence 


\section{INTRODUÇÃOO}

No campo da ciência, a relação histórica entre os Estados Unidos da América - EUA - e 0 Brasil, que se inicia no final do século XIX, consolida-se após a 2ª Guerra Mundial. Foi neste período que os rumos do campo científico brasileiro assumem, principalmente a partir dos anos de 1960, 0 ideal de ciência instituído nos EUA (ESCADA, 2010; JACOBS, 2004; DIAS, 2005). A aproximação entre os dois países fazia parte da política externa norte-americana do pós $2^{2}$ Guerra Mundial, com a promulgação de uma série de programas e ações bilaterais e multilaterais com países do Terceiro Mundo (RIBEIRO, 2006).

Um dos eventos marcantes dessa relação foi o processo de institucionalização da ciência, que se inicia em 1951, com a criação do Conselho Nacional de Desenvolvimento Científico e Tecnológico (CNPq), tendo como referência a agência norte-americana National Science Foundation - NSF (ANDRADE, 2001, ESCADA, 2010, JACOBS, 2004). Nesse mesmo ano, foi criada a Coordenação de Aperfeiçoamento de Pessoal de Nível Superior (CAPES), (ESCADA, 2010; MENDONÇA, 2000). Com a criação desses dois órgãos institucionais, CNPq e CAPES, as bases para a elaboração e a implementação de políticas para o setor científico e tecnológico estavam lançadas.

A constituição da relação bilateral entre Brasil e os EUA no campo científico influenciou na constituição simbólica do campo educacional no país. 0 principal lugar onde essa influência pode ser percebida é a Universidade, que passou a ser campo de formação de recursos para a produção de conhecimento científico e desenvolvimento tecnológico. O lugar social onde o capital científico passou a ser produzido e disputado entre seus agentes sociais.

Nesse cenário de influência norte-americana e consequentes mudanças no campo estrutural e simbólico ocorridas no Brasil, a Educação Física (EF) brasileira começou a dar os primeiros passos como área de produção de conhecimento científico e de formação de pessoal com alto grau acadêmico. E, a partir da década de 1980, desencadeou-se, uma série de debates com o objetivo de avançar na discussão sobre sua identidade acadêmico-científica. Parte significativa desse movimento, reconhecidamente, tomou como referência o "movimento disciplinar" ocorrido na EF norte-americana, a partir dos anos de 1960 (TANI, 1988, 1989, 1996; DIAS, 2005; SOUZA NETO, 1999; REIS; 2003; CAMPOS, 2003).

Franklin M. Henry, da Universidade da Califórnia, em Los Angeles, publica em 1964, no Journal of Health, Physical Education and Recreation, o artigo Physical education: an academic discipline, que desencadeou ações de valorização acadêmica da área, como uma reação à tentativa de excluí-la das universidades. $O$ questionamento ao status da EF no sistema universitário norteamericano foi respondido com argumentos que procuravam demonstrar que a área constituía-se numa disciplina acadêmica.

Entretanto, não é possível identificar nas discussões presentes na EF brasileira uma busca pela compreensão do cenário científico e político dos EUA na qual a EF norte-americana estava inserida. Os estudos e propostas de organização acadêmica da EF brasileira, a partir do referencial norte-americano, assumem como referência as discussões sobre o movimento disciplinar deflagradas por Henry como sendo um fato único, destituído de contexto, sem uma análise mais apropriada do que está na base do "movimento disciplinar" na EF norte-americana.

Identificar o capital valorizado no campo científico, compreendê-lo como parte integrante de um todo e estabelecer as posições na estrutura social é fundamental para sabermos, com 
maior propriedade, como se deu o processo/proposta de constituição do campo acadêmicocientífico da EF nos EUA. Pois, conforme afirma Bourdieu (2007, p. 31), se "é verdade que 0 real é relacional, pode acontecer que eu nada saiba de uma instituição acerca da qual julgo saber tudo, porque ela nada é fora das suas relações com o todo".

Nesse sentido, as trilhas percorridas, como a historicidade da Ciência e da EF nos EUA, foram fundamentais para que se pudesse contar e recontar alguns feitos, reconhecendo neles as rupturas e estruturas fundamentais do pensamento científico norte-americano. Adotamos como hipótese que os desdobramentos ocorridos na EF nos EUA podem ser descritos como ações para se apropriar das novas regras que passaram a orientar o campo científico norteamericano nesse período, com grande impacto político para a organização acadêmica.

O objetivo desse ensaio foi identificar como alguns elementos da organização política do campo científico nos EUA podem ter influenciado a organização da área de conhecimento da EF norte-americana a partir da década de 1960. Para tanto, agentes e eventos históricos significativos no campo científico e político são destacados e, consequentemente, suas influências na estrutura e organização e do campo da EF nos EUA.

\section{CENÁRIO CIENTÍFICO NORTE-AMERICANO: O VALOR SIMBÓLICO DA CIÊNCIA APÓS A 2 a GUERRA}

Durante a guerra, a pesquisa científica e tecnológica converteu-se em fonte de geração de novas tecnologias (MOREIRA; VELHO, 2008). A ciência e o discurso científico tornaram-se poderosos recursos político e diplomático de desenvolvimento econômico e social (ESCADA, 2010; FAGUNDES, 2009). Nesse cenário de valorização da ciência, em 1945, o presidente dos EUA, Franklin Delano Roosevelt, encomendou a elaboração de uma proposta para fortalecer o papel da ciência (STOKES, 2005).

$\mathrm{Na}$ Carta que Roosevelt enviou para Vannevar Bush, diretor do Office of Scientific Research and Development (OSRD), havia quatro pontos norteadores: 1) a segurança militar; 2) a guerra da ciência contra as doenças; 3) o papel do governo no apoio às atividades de pesquisa em instituições e organizações públicas e privadas, e; 4) a possibilidade de propor um programa eficaz para a descoberta e o desenvolvimento de talentos científicos na juventude.

O documento "Science, the Endless Frontier", Relatório Bush, estreitou a relação entre governo e ciência ao apontar o Estado como principal investidor em ciência básica e a universidade como campo para sua realização. A proposta era transformar os EUA em país independente na produção de pesquisa básica no campo da ciência, pois para Bush (1945, p. 6), "pesquisa científica básica é capital científico".

Para Bourdieu (2004), a composição da estrutura de grupos sociais depende do volume de capital científico acumulado por esses grupos de agentes. Assim, ciência básica e a Universidade passam a serem interesses fundamentais e o financiamento do Estado passa a ser um objeto de disputa entre grupos. Com o acúmulo dessas propriedades específicas, é constituído o capital científico e os Estados Unidos começam a traçar sua hegemonia no campo científico internacional, investindo na aquisição de volume de capital científico.

O foco principal de Bush na elaboração do relatório era a manutenção do apoio governamental às investigações científicas de natureza básica. Para tanto, tinha como proposta 
a criação da "National Research Foundation - NSF - com responsabilidades em relação à pesquisa básica tão ampla quanto as do OSRD durante a guerra" (STOKES, 2005, p. 84). A NSF foi o marco da institucionalização da ciência nos EUA, desenvolvendo políticas para a educação e a pesquisa, oferecendo suporte para a ciência básica e estimulando o talento científico de jovens por meio de bolsas de estudo. (STOKES, 2005; BUSH, 1945).

São duas as ideias centrais contidas no Relatório Bush, que marcaram as relações entre governo, ciência e tecnologia: pesquisa básica e desenvolvimento tecnológico (BUSH, 1945; STOKES, 2005; DIAS, 2005). Primeiramente, a pesquisa básica é colocada como essencial para que os Estados modernos atinjam seus objetivos nacionais; a segunda, defende que o conhecimento gerado pela pesquisa básica percorre uma trajetória linear até culminar na inovação tecnológica. Buscava-se enfatizar que, em países onde não existem políticas de Estado à ciência, o desenvolvimento econômico e social seria mais lento (FAGUNDES, 2009; DIAS, 2005).

A vertente ideológica da ciência básica do Relatório Bush fez com que o campo científico nos EUA se (re)organizasse de tal maneira que somente a crença de que pesquisa básica, livre, realizada sem fins práticos e utilitários, poderia ser geradora de progresso tecnológico. Com o Relatório Bush, um novo paradigma científico emergiu a partir da distinção significativa entre ciência básica e ciência aplicada, criando uma relação de dependência da segunda para com a primeira (DIAS, 2005; STOKES, 2005; BUSH, 1945).

Os fundamentos ideológicos do relatório Bush e sua principal iniciativa organizacional, a criação de uma National Science Foundation, ficaram em discussão no Congresso Nacional por cinco anos, entre 1945 e 1950. E, com o aprofundamento da crise de relações entre os EUA e o bloco Soviético, durante a Guerra Fria, fez-se emergencial a necessidade de colocar em prática a criação da NSF (MACILWAIN, 2000).

\section{A OBJETIVAÇÃO DAS MUDANÇAS NO CAMPO DA CIÊNCIA E DA EDUCAÇÃO}

O lançamento do Sputnik I, em 1957, "tornou claro quão profundamente o paradigma de Bush estava embebido na consciência da comunidade de políticas" (STOKES, 2005, p. 92), que reagiu a este acontecimento como um desafio ao campo da ciência e dos avanços tecnológicos alcançados.

No campo educacional e científico, o Sputnik é considerado o responsável por trazer apoio federal à investigação científica, pós-graduação, construção de edifícios e laboratórios (GEIGER, 1997), levando a ciência nos EUA a uma necessidade de maior apoio do Estado. (WANG; ORESKES, 2008). Nesta perspectiva de "reação" ao Sputnik, no campo da educação, o Estado deu um primeiro passo significativo com a aprovação do National Defense Education Act of 1958 - NDEA (Ato Nacional em Defesa da Educação (FOOTLICK, 1968; GEIGER, 1997; FLATTAU et al., 2006).

Abrangendo toda a estrutura do sistema educacional, o NDEA foi fundamental por criar o esboço tanto de um instrumento legal para a criação dos alicerces e o fortalecimento da pesquisa básica nas universidades e também por materializar um princípio ideológico reformulador da educação pública, preconizado por James Briant Conant (1953). Conant $(1953,1963)$ afirmava que se a educação pública era tão importante para a nova realidade 
do país, em plena Guerra Fria, os homens da ciência deveriam ajudar as escolas públicas a desempenharem um papel tão significativo como certos físicos e químicos durante a $2^{\underline{a}}$ Guerra Mundial. Buscava alinhavar os avanços da ciência ocorridos nas universidades com os cursos de formação de professores, pretendendo introduzir nestes cursos, assim como nos currículos das escolas básicas, um caráter mais científico e menos pedagógico.

Para esse pensador, a relação entre os saberes presentes nas disciplinas científicas e as escolas parecia ser o caminho para melhorar a formação docente (CONANT, 1953). As universidades foram afetadas por estes ideais e as áreas que sofreram críticas foram justamente os departamentos de educação e cursos de formação de professores, por seu caráter pedagógico, não mais compreendido como modelo ideal (CONANT, 1953; FOOTLICK, 1968).

Outra proposta apresentada pelo NDEA era o aumento na concessão de bolsas de estudo para estudantes de pós-graduação (FLATTAU et. al., 2006), na tentativa de melhorar a qualificação dos professores universitários, aumentando a quantidade de professores doutores e tornar mais equânime a sua distribuição geográfica.

Se o NDEA foi o primeiro avanço legal/institucional na relação entre Estado, Ciência e Universidade, orientado pelo paradigma científico de Vannevar Bush, essa relação objetivouse no documento publicado em 1960, Scientific Progress, the Universities, and the Federal Government, conduzido pelo presidente do Panel on Basic Research and Graduate Education, Glenn T. Seaborg, (NATIONAL SCIENCE FOUNDATION, 1960; GEIGER, 1997; SEABORG, 1990, 1992).

O Seaborg Report, como ficou conhecido, consolidou a concepção de ciência voltada para a produção da pesquisa básica e reforçou a responsabilidade do Estado na distribuição de recursos para a produção de conhecimento básico realizado na pós-graduação (GEIGER, 1997; DOUGLASS, 1999), afirmando que a quantidade e a qualidade da pesquisa e da pós-graduação dependeriam das ações do governo (PRESIDENT'S SCIENCE ADVISORY COMMITTEE, 1960).

Este documento foi impactante por fortalecer quatro elementos estruturantes do campo científico: a) aumento dos investimentos em ciência básica; b) pós-graduação como lugar de produção da pesquisa básica; c) o fortalecimento da ciência para o bem-estar da nação e; d) a parceria entre Universidade e Governo como a base para a obtenção de uma ciência de ponta (GEIGER, 1997).

\section{CAMPO DA EDUCAÇÃO FÍSICA DIANTE DAS MUDANÇAS DO CAMPO EDUCACIONAL E CIENTÍFICO}

A Educação Física (EF), inserida no mundo da Universidade, não estava alheia às mudanças ocorridas no campo científico, educacional e político. Na busca por compreender como esta reestruturação influenciou as mudanças na área, destaca-se o estado da Califórnia como cenário principal por três aspectos: (a) aprovação do Master Plan for Higher Education in California (1960-1975); (b) aprovação do Fisher Act, de 1961 e; (c) o início do "Movimento Disciplinar" deflagrado por Franklin Henry, em 1964.

A Califórnia era um estado avançado nas questões de políticas educacionais, sendo modelo para os demais (FOOTLICK, 1968). A partir do momento em que o governo federal assume 
o papel financiador da pesquisa básica e da pós-graduação nas universidades, a Califórnia viu a necessidade de estabelecer critérios para definir quais instituições receberiam investimentos.

A principal política elaborada para nortear o funcionamento do campo acadêmico e científico na Califórnia foi o Master Plan for Higher Education in California, que vigorou de 1960 a 1975 e apontou a necessidade da diferenciação funcional entre os cursos de graduação das "state colleges" e os demais oferecidos nas universidades. Um dos motivos foi a crescente preocupação com a competição por recursos financeiros entre os "state colleges" e a University of Califórnia (CALIFORNIA STATE DEPARTMENT OF EDUCATION, 1960, p. xi).

0 documento estabeleceu três estratificações entre as instituições de ensino superior no estado: a) junior colleges - responsáveis pela realização de cursos técnicos, com formação em até 02 anos; b) state colleges, para currículos ocupacionais, com cursos de formação em até 04 anos; c) e o sistema da University of Califórnia, onde ficaria concentrada as funções da educação profissional, pesquisa e pós-graduação (CALIFORNIA STATE DEPARTMENT OF EDUCATION, 1960; FOOTLICK, 1968).

Ao determinar o desenvolvimento do campo da pesquisa e pós-graduação como responsabilidade da Universidade, os programas de doutorado passaram a ser prioritários na distribuição de recursos. Nesse sentido, o Master Plan estabeleceu a Universidade como autoridade única no ensino superior público para atribuição do grau de doutor. (CALIFORNIA STATE DEPARTMENT OF EDUCATION, 1960).

Outro evento histórico ocorrido na Califórnia e que atingiu diretamente a EF foi a Licensing and Certificated Personnel Act (Lei de Licenciamento e de Pessoal Certificado), de 1961, conhecido como Fisher Act. O Fisher Act foi uma resposta do Estado da Califórnia a uma crescente insatisfação nacional com o currículo da escola pública e com a formação de professores (INGLIS, 1961). Este autor deixa isso claro quando fala que os educadores da Califórnia acreditavam que o processo de credenciamento dos professores era muito complexo e que também havia o desejo, por parte de alguns professores universitários e cidadãos, para retornar a uma ênfase mais acadêmica no ensino em escolas públicas (INGLIS, 1961; 1965).

Portanto, o objetivo desta lei foi a criação de novos critérios para a formação de professores, priorizando o fortalecimento da formação acadêmica e a necessidade de estabelecer um sistema de certificação e credenciamento para poder atuar nas escolas públicas (INGLIS, 1961; 1965). As principais propostas presentes no Fisher Actforam: (a) os requisitos voltados à preparação acadêmica aumentaram em detrimento da preparação profissional; (b) a formação para o ensino no contexto escolar foi limitada às áreas de preparação acadêmica; (c) propôs-se a distinção entre a preparação acadêmica e não-acadêmica; (d) os professores do ensino fundamental deveriam ter cinco anos de preparação para ser considerada uma formação acadêmica e; (e) a preparação em um campo acadêmico passaria a ser condição obrigatória para os administradores escolares (BROTT, 1984).

Os pontos do Fisher Act que apresentaram maior divergência, debatidos pelos legisladores, sociedade civil e grupos profissionais, estavam na deliberação sobre os critérios que orientariam a divisão entre o que seria uma área/curso acadêmico e não-acadêmico. Porém, não ficou uma definição clara na lei dos termos acadêmico e não-acadêmico, o que dificultou a compreensão do que seria um curso acadêmico. Todavia, é possível pensar que mesmo não estando explicitada, supõese que uma disciplina acadêmica se sustentaria com produção científica nos moldes da ciência hegemônica: ciência básica (BROTT, 1984). 
Segundo Inglis (1961), o problema que recaía sobre a EF era o fato de que existiam muitos administradores de escolas com formação em EF e uma das maiores mudanças da lei foi a exigência de credencial em graduação com grau acadêmico para atuar na administração das escolas públicas. A mudança foi planejada para reduzir a predominância de professores de EF exercendo funções administrativas, como a direção escolar (KROLL, 1982; INGLIS, 1961; 1965).

Ressaltamos que a EF não estava sendo considerada área acadêmica por não demonstrar, historicamente, ser uma área que tinha seu processo de formação profissional orientado pelos princípios do conhecimento científico e por ser uma área que não desenvolvia pesquisa de natureza básica (INGLIS, 1961). Logo, deixaria o sistema universitário e passaria a fazer parte das escolas superiores de formação profissional com quatro anos de duração (INGLIS, 1961), responsável apenas pela preparação de professores para atuarem no ensino fundamental (HENDRICK, 2002). Estava em jogo a permanência da EF no sistema universitário, pois, se uma área de formação superior ficasse fora deste grupo caracterizado como acadêmico-científico, reduziria sua capacidade de captar fomento para o crescimento e desenvolvimento da área por não possuir capital científico.

Alguns cursos que não apresentavam os requisitos acadêmicos se organizaram a partir de suas associações representativas de classe, estando entre eles a EF. A California Association for Health, Physical Education and Recreation (CAHPER) demonstrou uma visão oposta ao Fisher Act, defendendo que a EF era um curso acadêmico.

A EF foi um dos poucos cursos que conseguiu, após longo debate junto à Câmara Estadual de Educação, entre 1963-1965, alterar a sua posição, até então colocada como área não-acadêmica. Esse entendimento sobre a EF como área acadêmica não pode ser generalizado. Somente um curso foi enquadrado dentro da perspectiva acadêmica: o curso de EF da University of California. Este feito só foi possível pelo fato da justificativa apresentada pela CAHPER demonstrar que sua estrutura curricular continha conhecimento de base acadêmica da área das ciências biológicas (INGLIS, 1965), ancorado, fundamentalmente, nas disciplinas de aprendizagem motora, fisiologia do exercício e biomecânica (BAIN; POINDEXTER, 1981).

Nesse período de grandes discussões sobre o futuro da área, detonadas pelo Fisher Act , a publicação em 1963 do livro de James Bryant Conant, The Education of American Teachers, é considerada um dos eventos de maior impacto no campo da EF (ZEIGLER, 1982, LAWSON, 1985). Conant traz como foco de seu trabalho a educação elementar e secundária nos EUA e a formação de professores. Seu interesse pela educação norte-americana era saber se havia ou poderia haver uma educação científica em todo sistema educacional (CONANT, 1968).

Conant (1963) faz recomendações de caráter geral e específico na tentativa de que fosse realizada uma mudança qualitativa na formação de professores em diversas áreas. Chegou a classificar alguns cursos como medíocres, monótonos, desorganizados. Algumas destas recomendações diziam respeito justamente à EF, Língua Estrangeira e Música. No caso da EF, Conant (1963) propôs que as universidades deveriam cancelar os programas de pós-graduação, afirmando que a EF não possuía objeto de estudo, metodologia e um corpo de conhecimento. Logo, não possuía os elementos fundantes de orientação acadêmica, não devendo permanecer nas universidades (CONANT, 1963; BROEKHOFF, 1982). 
Este apontamento desestruturou o mundo de verdades existentes na área (ROSE, 1986), como a formação voltada para o campo profissional, de natureza prática e com objetivos diversos. Mas, se este evento causou forte impacto na EF, também fortaleceu a necessidade de reflexões sobre seu corpo de conhecimento. Porém, o campo da EF já estava se organizando e buscando se reestruturar a partir das novas regras e estruturas determinadas pelo campo científico e educacional.

Diante dos acontecimentos que atingiam a área da EF, algumas ações foram tomadas. Em 1964, o presidente da National College Physical Education Association for Men (NCPEAM), John E. Nixon (NATIONAL ASSOCIATION OF KINESIOLOGY AND PHYSICAL EDUCATION IN HIGHER EDUCATION, 2011), juntamente com a American Association for Health, Physical Education and Recreation (AAHPER), enviou para James B. Conant uma cópia da edição da Research Quarterly, publicada em maio de 1960 (KROLL, 1982). Os agentes e instituições tentavam convencer Conant de que os programas de pós-graduação da área produziam conhecimentos científicos e as suas críticas não tinham fundamento.

Autores como Park (2005) e McCristal (1969) também apontam que a EF, naquele período, não estava cega diante das mudanças no cenário científico e nas universidades, pois "as questões de como criar uma área acadêmica que fosse composta por aspectos relevantes das ciências biológicas, psicológicas e ciências sociais estavam fortemente presentes" (PARK, 2005, p. 16), com conhecimentos sendo produzidos, em todas suas disciplinas, desde a década de 1950 (McCRISTAL, 1969).

A EF foi levada à necessidade de aderir ao processo pelo qual o conhecimento estava sendo produzido. No entanto, esse processo perpassava pela combinação de "velhas" disciplinas, como vinha ocorrendo com outras áreas de conhecimento (PARK, 2005, McCRISTAL, 1969, KROLL, 1982, ROSE, 1986). E a EF, ao longo de sua história, baseou-se em disciplinas como psicologia, sociologia, fisiologia, filosofia, biologia e outras.

O problema apontado era que, historicamente, o profissional de EF tinha feito uso dos conhecimentos oriundos dessas disciplinas de forma pragmática (McCRISTAL, 1969, LAWSON; MORFORD, 1979; HENRY, 1978), com o interesse voltado para um saber prático, fornecendo justificativa para jogos, esportes e atividade física em geral. Como consequência, o desenvolvimento de novos conhecimentos era mais um acaso do que resultado de procedimentos teórico-metodológicos.

Mediante a necessidade de mudanças, movimentos e ações foram realizados por entidades representativas e universidades na década de 1960. Conforme Park (2005), no início dos anos de 1960 a College Physical Education Association - CPEA (Associação das Faculdades de Educação Física) - desencadeia um processo reflexivo sobre a EF. Joy Kistler, presidente da CPEA, em seu discurso oficial, em 1961, "Futuras Direções para a Educação Física", se referiu à crescente competição política, econômica e científica entre as nações, e que havia a necessidade de incutir nos estudantes de graduação e de pós-graduação em EF as habilidades necessárias para resolver problemas de grande complexidade científica.

Em 1962, a Big Ten Universities, um comitê formado por membros dos departamentos dos cursos de EF das Universidades de Indiana, lowa, Michigan, State Michigan, Minnesota, Northestern, Ohio State, Purdue, Wisconsin e Illinois, responsáveis por organizar os encontros anuais da Western Conference of Physical Education Directors (WCPED), e estabelecem como 
temática central dos encontros "The Body of Knowledge in Physical Education" (ZEIGLER; McCRISTAL, 1967).

A principal contribuição deste encontro foi a criação do Body of Knowledge Project, que tinha como foco organizar as discussões que já ocorriam em nível nacional sobre o corpo de conhecimento da $E F$, principalmente no campo da pós-graduação. A preocupação com a pós-graduação estava evidenciava, pois era justamente este campo que poderia garantir a permanência da EF, como disciplina científica, nas universidades.

De acordo com McCristal (1969) o Body of Knowledge Project seria a realização de uma série de simpósios de natureza acadêmica com a finalidade de desenvolver os conceitos científicos básicos da EF e qualificar os programas de pós-graduação na área. A realização desses eventos foi um esforço para proporcionar uma oportunidade de alto nível de capacitação para os "professores de pós-graduação melhorar a sua compreensão e aguçar suas técnicas de pesquisa em desenvolvimento de nosso corpo de conhecimento" (ZEIGLER; McCRISTAL, 1967, p. 84).

Segundo Zeigler e McCristal (1967), durante os encontros anuais da Big Ten nos anos de 1964 e 1965, publicações de artigos, palestras e conferências procuraram mostrar que a EF constituía-se uma disciplina acadêmica por possuir um corpo de conhecimento que estava presente na área do esporte, do movimento humano, da cinesiologia, do desempenho humano, da aprendizagem motora e outras.

A Big Ten, como resultado desse processo, apontou que o corpo de conhecimento da EF já estava, historicamente, presente nos currículos das instituições que a compunham. Este corpo de conhecimento seria utilizado para organizar e compor o currículo da EF na Educação Básica, Programas de Graduação e Pós-Graduação.

A materialização desta noção disciplinar ocorreu em agosto de 1966, com a definição das seguintes subáreas de especialização na EF: Fisiologia do Exercício, Biomecânica, Aprendizagem Motora, Psicologia do Esporte, Sociologia do Esporte, História, Filosofia, Teoria Administrativa e comparação entre Educação Física e Esporte (ZEIGLER; McCRISTAL, 1967).

Juntando-se a estes fatos de abrangência nacional e às leis estaduais que estavam em discussão e, principalmente, como uma resposta às críticas feitas por James $\mathrm{B}$. Conant à EF, Franklin Henry (1964), professor da UC-Berkeley, apresenta o texto "Physical Education - An Academic Discipline", no encontro anual da National College Physical Education Association for Men.

Henry (1964) enfatizou que uma disciplina acadêmica seria um corpo organizado de conhecimento e que a produção do que ele chamou de "conhecimento teórico" deveria ser desvinculada da aplicação prática. Também mencionou que havia um campo acadêmico de conhecimento básico da EF, constituído na relação com as seguintes áreas: anatomia, física, fisiologia, antropologia cultural, história e sociologia, e psicologia.

Assim, a área da EF seria composta por: (1) cinesiologia e biomecânica; (2) fisiologia do exercício; (3) ambiente e treinamento; (4) coordenação neuromotora; (5) senso cinestésico; (6) aprendizagem e transferência motora; (7) fatores emocionais e de personalidade no desempenho físico; (8) a relação de todos estes conhecimentos para o desenvolvimento humano, e; (9) o status funcional do indivíduo e sua habilidade no envolvimento na atividade 
motora. Com sua proposta, Henry (1964, p. 17) defendeu que "se a disciplina acadêmica da EF ainda não existe, seria necessário inventá-la".

\section{CONSIDERAÇÕES FINAIS}

Ao construirmos a temática e o processo investigativo dos elementos norteadores da organização do campo científico nos EUA e o seu impacto na organização da área de conhecimento da EF, proposta na década de 1960, foi necessário lançarmos um olhar sobre a maneira como se deu a inserção da EF em um complexo cenário de trocas simbólicas sobre o qual a EF não tinha poder (capital político e científico), para determinar as regras do que seria (ou não) científico.

Foi a partir de tomadas de decisões políticas, traçando o futuro do campo científico, visando estabelecer um lugar social para a realização da pesquisa "livre e geradora de conhecimento básico" que a universidade se tornou, de forma legítima, o campo de produção de conhecimento. Assim, as disciplinas que pudessem dar sua contribuição ao desenvolvimento do conhecimento teriam um lugar no topo desta estrutura e poderiam compartilhar de um status único: o do avanço do conhecimento que levaria ao desenvolvimento e estabelecimento de um país como potência mundial.

$A E F$, frente aos grupos de maior capital científico e às novas regras do campo da ciência, buscou criar suas estratégias de luta, alterando as regras em seu próprio campo social. Compreendemos que as ações e o discurso científico adotado pela EF foram estratégias empregadas para se apropriar das novas propriedades que estavam constituindo o campo da ciência nos EUA após a 2 ${ }^{\underline{a}}$ Guerra Mundial.

Acreditamos que o movimento disciplinar sofreu pressões do campo científico e se reorganizou dentro dos moldes do que se esperava que fosse uma área de conhecimento científico. A estratégia foi investir na caracterização acadêmica dos cursos de graduação e pós-graduação, adotando a produção científica como pilar da identidade acadêmica. Com isso, os grupos que apresentavam condições de produção científica próximas do ideal de ciência (ciência básica, ciências naturais, quantificação e rapidez na disseminação do conhecimento) se sobressaíram na estrutura social do campo científico da EF norte-americana.

A relevância histórica atribuída ao texto de Henry (1964) ocorre por estruturar sua proposta de disciplina acadêmica da EF a partir: a) das discussões que estavam ocorrendo no campo científico norte-americano e no interior do próprio campo da EF (como a aproximação com disciplinas de maior capital científico), e; b) dos elementos fundantes do ideal de ciência estabelecidos no pós 2 ${ }^{\mathrm{a}}$ Guerra Mundial e difundidos pelo Relatório Bush.

Franklin Henry construiu seu discurso propositivo para uma disciplina da EF a partir do ideal científico que norteou as críticas à EF: o modelo linear de ciência básica. A (re)estruturação do campo científico na EF norte-americana se deu a partir de um cenário de mudança de paradigma científico. $\mathrm{O}$ ideal de Henry para a constituição da disciplina acadêmica da EF norte-americana estava influenciado pelo ideal científico presente naquele momento histórico e ancorado em propostas que sistematizavam anseios, discussões e ideais que emergiam tanto do campo acadêmico como do campo profissional. Nesse sentido, não mais podemos atribuir a Conant (1693), e à resposta dada a ele por Henry (1964) como sendo fatores únicos e lineares 
que tenham desencadeado toda a discussão sobre a constituição de uma área acadêmica da EF nos EUA.

\section{REFERÊNCIAS}

ANDRADE, Ana Maria Ribeiro. Ideais políticos: a criação do conselho nacional de pesquisas.

Revista Parcerias Estratégicas, Brasília, v.11, p. 221-242, jun. 2001.

BAIN, Linda; POINDEXTER, Hobert B.W. Applying disciplinary knowledge in professional preparation. Journal of Physical Education, Recreation and Dance, Reston, v. 52, p. 40-44, 1981.

BOURDIEU, Pierre. O poder simbólico. 11. ed. Rio de Janeiro: Bertrand Brasil, 2007.

BOURDIEU, Pierre. Os usos sociais da ciência: por uma sociologia clínica do campo científico. São Paulo: EDUNESP, 2004.

BROEKHOFF, Jan. Physical education as a profession. Quest, Champaing, n. 31, p. 244-254, 1979.

BROTT, Ralph. Credentialism in California: alternatives for governance. Berkeley: University of California, 1984.

BUSH, Vannevar. Science, the endless frontier. Washington, 1945. Disponível em: <http:// www.nsf.gov/od/lpa/nsf50/ vbush1945.htm>. Acesso em: 9 set. 2009.

CALIFORNIA STATE DEPARTMENT OF EDUCATION. A master plan for higher education in California (1960-1975). Sacramento: California State Printing Office, 1960. Disponível em: $<$ http://www.ucop.edu/ acadinit/mastplan/MasterPlan1960.pdf>. Acesso em: 9 set. 2009.

CAMPOS, Adriano Pires. A construção do campo acadêmico da educação física brasileira: análise da Escola de Educação Física e Esporte da Universidade de São Paulo. 2003. Dissertação (Mestrado em Ciências da Motricidade) - Instituto de Biociências, Universidade Estadual Paulista, Rio Claro. 2003.

CONANT, James Bryant. Como compreender a ciência. São Paulo: Cultrix, 1964.

CONANT, James Bryant. Dois modos de pensar meus encontros com a ciência e a educação. São Paulo: Nacional, 1968.

CONANT, James Bryant. La educación en un mundo dividido: función de las escuelas públicas en nuestra sociedad. Buenos Aires: Nova, 1953.

CONANT, James Bryant. The education of American teachers. New York: McGraw-Hill, 1963.

DIAS, Rafael Brito. A política científica e tecnológica latino-americana: relações entre enfoques teóricos e projetos políticos. 2005. 106 f. Dissertação (Mestrado em Política Científica e Tecnológica) - Universidade Estadual de Campinas, Campinas. 2005

DOUGLASS, Jhon Aubrey. The cold war, technology and the American University. UC Berkeley: Center for Studies in Higher Education. 1999. Disponível em: <http://escholarship.org/ uc/item/9db970dq>. Acesso em: 6 jul. 2010. 
ESCADA, Paulo Augusto Sobral. Construção e usos sociais da pesquisa científica e tecnológica: um estudo de caso da divisão de processamento de imagem do INPE. 2010. 231 f. Tese (Doutorado em Ciência Política) - Faculdade de Filosofia, Letras e Ciências Humanas, Universidade de São Paulo, São Paulo, 2010.

FAGUNDES, Ailton Laurentino Caris. Caminhos que se bifurcam: idéias, atores, estratégias e interesses na política científica e tecnológica do regime militar. 2009. 261 f. Tese (Doutorado em Ciência Política) - Faculdade de Filosofia, Letras e Ciências Humanas, Universidade de São Paulo, São Paulo, 2009.

FLATTAU, Pamela Ebert et al. The National Defense Education Act of 1958: selected outcomes. 2006. Disponível em: <http://www.nagc.org/uploadedFiles/Information and Resources/ Hot Topics/The\%20National\%20Defense\%20Act.pdf>. Acesso em: 5 maio 2010.

FOOTLICK, Jerrold. Uma nova era para a educação. Rio de Janeiro: Bloch, 1968.

GEIGER, Roger. Research \& relevant knowledge: American research universities since world war II. 2. ed. New York: Oxford University, 2009.

GEIGER, Roger. What happened after sputnik? Shaping university research in the United States. Minerva, Netherlands, n. 35, p. 349-367, 1997.

HENDRICK, Irving. Establishing state responsibility for the quality of teachers: 1850-1952. 2002. Disponível em: <http://www.ctc.ca.gov/commission/history/1850-1952-Est.pdf>. Acesso em: 10 out. 2009.

HENRY, Franklin M. Physical education: an academic discipline. Journal Of Health, Physical Education, Recreation, Reston, v. 35. n. 7, p 32-38. 1964.

HENRY, Franklin M. The academic discipline of physical education. Quest, Champaing, n. 29, p. 13-29. 1978.

HOFFMAN, Shirl J. Specialization + fragmentation = extermination: A formula for the demise of graduate education. Journal of Physical Education, Recreation and Dance, Reston, v. 56, p. 19-22, 1985.

INGLIS, Sidney A. Specialized Interests challenge California Fisher act 1961-1965. Disponível em: <http://www.ctc.ca.gov/commission/h>. Acesso em: 20 nov. 2009.

INGLIS, Sidney A. The fisher reformation 1953-1961. Disponível em: <http://www.ctc.ca.gov/ commission/history/1953-1961-Fisher-Reform.pdf>. Acesso em: 20 nov. 2009.

JACOBS, Camila Campos. A participação da United States Agency for international development (USAID) na reforma da universidade brasileira na década de 1960. 2004. 152 f. Dissertação (Mestrado em Relações Internacionais) - Instituto de Filosofia e Ciências Humanas, Universidade Federal do Rio Grande do Sul, Porto Alegre, 2004.

KROLL, Walter. Graduate study and research in Physical Education. Champaing: Human Kinectics, 1982.

LAWSON, Hal A. Challenges to graduate education. Journal of Physical Education, Recreation and Dance, Reston, n. 53, p. 23-25, 1985.

LAWSON, Hal A; MORFORD, Robert. The cross disciplinary structure of kinesiology and sports studies: distinctions implications and advantages. Quest, Champaing, n. 31, p. 222-230, 1979. 
MACILWAIN, Colin. Revisiting the politics of American science. Minerva, Netherlands, v. 38, n. 3, p. 349-361, 2000.

McCRISTAL, King J. Body of knowledge symposiums. Journal of Health, Physical Education, Recreation, Reston, v. 40, n. 9, p. 20, 1969.

MENDONÇA, Ana Walesca P.C. A universidade no Brasil. Revista Brasileira de Educação, Rio de Janeiro, n. 14, p. 131-150, maio/ago. 2000.

MOREIRA, Maria Ligia; VELHO, Léa. Pós-graduação no Brasil: da concepção ofertista linear para novos modos de produção do conhecimento implicações para avaliação. Avaliação, Campinas, v. 13, n. 3, p. 625-645, nov. 2008.

\section{NATIONAL ASSOCIATION OF KINESIOLOGY AND PHYSICAL EDUCATION IN HIGHER} EDUCATION. National college physical education association for men (NCPEAM). 2011. Disponível em: <http://www.nakpehe.org/documents/NCPEAM.html>. Acesso em: 15 jan. 2011.

NATIONAL SCIENCE FOUNDATION. 10th Annual Report 1960. Washington-DC U.S.: Government Print Office. 1960. Disponível em: <http://pt.scribd.com/doc/1001611/National-ScienceFoundation-ar-1960>. Acesso em: 12 out. 2010.

PARK, Roberta. Of the greatest possible worth: the research quarterly in historical contexts. Research Quarterly for Exercise and Sport, Reston, v. 76, supl. n. 2, p. 5-26, 2005.

POLANYI, Michael. The republic of science: its political and economic theory. Minerva, Netherlands, v. 38, n.1, p. 1-32, 2000.

PRESIDENT'S SCIENCE ADVISORY COMMITTEE. Scientific progress, the universities, and the Federal Government; statement. Washington, DC, [U.S. Govt. Print. Off.], 1960.

REIS, Marize Cisneiros Costa. A legitimidade acadêmico-científica da Educação Física: uma investigação. 2003. 301 f. Tese (Doutorado em Educação Física) - Faculdade de Educação Física, Universidade Estadual de Campinas, Campinas, 2003.

RIBEIRO, Ricardo Alaggio. A aliança para o progresso e as relações Brasil- Estados Unidos. 2006. 375f. Tese (Doutorado em Ciência Política) - Instituto de Filosofia e Ciências Humanas, Universidade Estadual de Campinas, Campinas, 2006.

ROSE, David. Is there a discipline of physical education? Quest, Champaing, v. 38, n.1, p. 1-21, 1986.

SAGE, Georgeh; DYRESON, Marks; KRETCHMAR, Scottr. Sociology, history, and philosophy in the research quarterly. Research Quarterly for Exercise and Sport, Champaing, v. 76, supl. n. 2, p. 88-107, 2005.

SEABORG, Glenn T. Evolution of federal support of science. Washington-DC, 1990.

Disponível em: <http://www.osti.gov/bridge/servlets/purl/6557054-JDlw9b/>. Acesso em: 13 maio 2010.

SEABORG, Glenn T. Glenn T. Seaborg: autobiography. 1992. Disponível em: <http://www.lbl. gov/Publications/Seaborg/bio.htm>. Acesso em: 13 maio 2010.

SOUZA NETO, Samuel. A Educação Física na Universidade: licenciatura e bacharelado: as propostas de formação profissional e suas implicações teórico-práticas. 1999. 350f. Tese (Doutorado em Educação) - Universidade de São Paulo, São Paulo, 1999. 
STOKES, Donald E. O quadrante de Pasteur: ciência básica e inovação tecnológica. Campinas: Ed. da UNICAMP, 2005.

TANI, Go. Cinesiologia, educação física e esporte: ordem emanante do caos na estrutura acadêmica. Motus Corporis. Rio de Janeiro, v. 3, n. 2, p. 9-50. 1996.

TANI, Go. Perspectivas da Educação Física como disciplina acadêmica. In: SIMPOSIO PAULISTA DE EDUCACAO FISICA, 2, 1989, Rio Claro. [Anais...]. Rio Claro: UNESP, Departamento de Educação Física, 1989. p. 2-13.

TANI, Go. Pesquisa e pós-graduação em Educação Física. In: PASSOS, Solange Cássia Elias. (Org). Educação física e esporte na universidade. Brasília: SEED-MEC, 1988. p. 381-394.

WANG, Zuoyue; ORESKES, Naomi. History of science and American science policy. ISIS,Chicago, v. 99, n. 2, p. 365-373, 2008.

ZEIGLER, Earle. Physical education: dead, quiescent, or undergoing modification? Journal of Physical Education, Recreation and Dance, Reston, v. 53, p. 51-53, 1982.

ZEIGLER, Earle; McCRISTAL, King J. A history of the big ten body-of-knowledge project in physical education. Quest, Champaing, v. 9, n. 1, p. 79-84, 1967. 